\title{
Liquid-liquid secondary fragmentation with solidification
}

\author{
M. Hadj-Achour, N. Rimbert*, M. Gradeck \\ Laboratoire d'Énergétique et de Mécanique Théorique et Appliquée (LEMTA), Lorraine \\ University-CNRS, France \\ *Corresponding author: nicolas.rimbert@univ-lorraine.fr
}

\begin{abstract}
In the event of a hypothetical core disruptive accident in nuclear power plants, the molten core may flow out the reactor vessel and interact with the cold water. The evolution of the accident is strongly affected by the fragmentation of the jet of molten metallic fuel due to its interaction with the water (i.e. this situation is known as fuel coolant interaction, $\mathrm{FCl}$ ). In order to evaluate and predict the various consequences of a $\mathrm{FCl}$, many researches are conducted with either corium or high melting temperature molten metal, where premixing stage evolves with an important production of steam. This steam production that is unavoidable because the high temperature of corium leads to difficulties for using optical diagnostics. Hence, in our case, we use a eutectic alloy (Field's metal) with a low melting point $\left(62^{\circ} \mathrm{C}\right)$ in order to be able to visualize correctly the droplet fragmentation processes.
\end{abstract}

The present work focuses on the fragmentation of a single Field's metal liquid droplet with mass equals to $0.27 \mathrm{~g}$ $( \pm 0.01 \mathrm{~g})$. The liquid droplet interacts with a water pool whose temperature range between $20^{\circ} \mathrm{C}$ to $60^{\circ} \mathrm{C}$. According to its Weber number, it fragments in different ways. For each experiment, a single droplet has been visualized using a high-speed camera (at $8000 \mathrm{fps}$ ). All measurements (drop size, velocity, impact parameter and geometrical properties of the drops after the penetration) into the pool are evaluated using an open source image processing. Solidified fragments can then be sieved and the size PDF determined. Focus of the present work is put on the evolution of the Sauter Mean Diameter with increasing Weber number and varying bath pool temperature. It is shown that using a simple crust model during solidification and defining an effective Weber number which include the crust elasticity all the curves collapse on the same master curve for all the water bath temperature considered.

\section{Keywords}

Liquid Metal Spray, Nuclear Safety, Powder production, Fragmentation, Solidification

\section{Introduction}

One of the worst sequels of nuclear accident is nuclear core meltdown. In the event of primary containment vessel failure, the melted core (or corium) can pour outside and interact with the surrounding water (presence of surrounding water is uncertain and is related to the handling of the accident). The size and the distribution of the resulting fragments is still a scientific open problem. Yet it may be related to atomization and spray studies and to the field of metallic powder production as the interplay between fragmentation and solidification is a common mechanism. Present work focuses on what is traditionally called secondary atomization or more exactly to the fragmentation mechanism of a single droplet in a surrounding flow field. However, the problem will be simplified as vapour production (which may result in a vapour explosion in the hottest cases and prevent using simple optical diagnostics) will be prevented. Therefore present work will be quite close to previous studies by Hsiang and Faeth [1], Landeau et al. [2] or Ranger and Nicholls [3] for instance. However these works focus on either very high or very low density ratio, whereas for the intermediate density ratio (around ten) results are scarcer (see Gelfand [4], Kim et al. [5] for instance). Present work will therefore focus on the fragmentation and solidification mechanisms of a mildly hot single liquid metal droplet in water with a density ratio between phases close to 8 .

Fragmentation mechanisms are strongly impacted by value of the Weber, Ohnesorge and Reynolds numbers and fragmentation regimes are determined as a function of these numbers:

$$
W e=\frac{\rho_{A} D_{0} U_{L}^{2}}{\sigma}, O h=\frac{\mu_{L}}{\sqrt{\sigma \rho_{L} D_{0}}}, \operatorname{Re}=\frac{\rho_{A} D_{0} U_{L}}{\mu_{L}} .
$$


However, in order to take into account solidification during fragmentation, an apparent Weber number is proposed, following Haraldsson [6]. In this model surface tension is increased during solidification to take into account the elasticity of the solidified crust. Thought initially proposed to modify Kelvin-Helmholtz during solidification, it will be shown that it can also be used to propose a correlation for the Sauter Mean Diameter of the solidified fragments as a function of this new Weber number.

As present work is dominantly experimental, the experimental setup will be detailed and results discussed but a comparison with recent results obtained using Direct Numerical Simulation with the Open Source Code Basilisk (cf. Popinet [7]) will illustrate the similarity between experiments and simulation.

Last section will present how introducing a modified Weber number leads to an efficient data reduction.

\section{Material and methods}

Experimental setup is detailed on figure 1, a droplet generator is located above a 500 L liquid water pool that can be maintained at a given temperature thanks to a plunging heating element. Measurements are made using shadowgraphy thanks to a LED projector and a high speed camera. Solidified fragments are then collected (after several hundredth droplet impingements) and sieved to obtain the fragments size distribution. The liquid metal used in present experiments is Field's metal whose properties are given in Table 1. It is a eutectic alloy of Tin, Bismuth and Indium whose melting temperature is $62^{\circ} \mathrm{C}$. Therefore dropping $85^{\circ} \mathrm{C}$ liquid metal droplets into water does not produce any vapour.

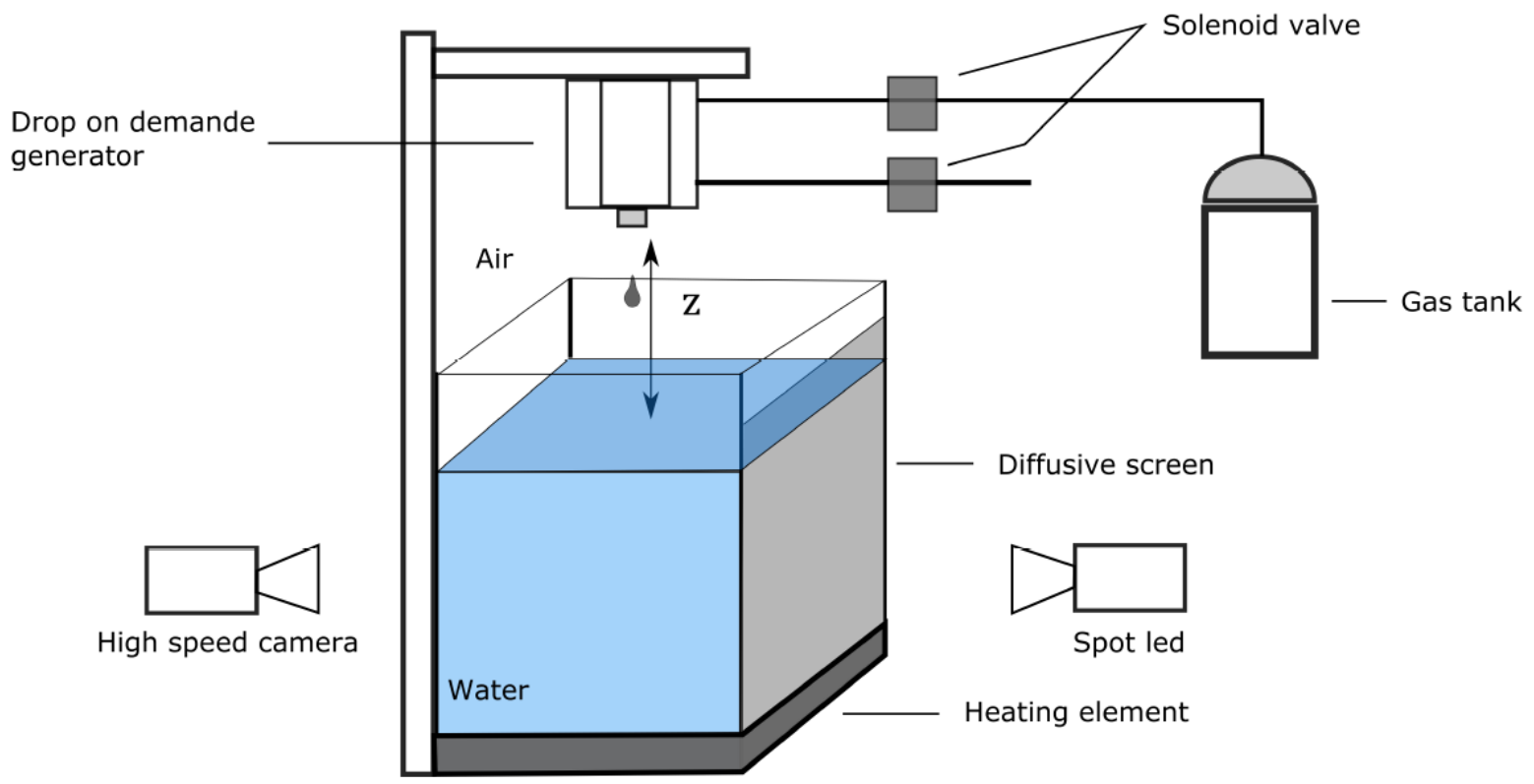

Figure 1. Picture of the experimental setup.

Liquid metals have commonly a very high surface tension, $\sigma=0.4 \mathrm{~N} / \mathrm{m}$, in the present case. However they are very sensitive to oxidation, cf. Xu et al. [8]. A thin oxide layer can appear that gives them yield stress rheological behavior. Therefore their initial shapes are close to yield stress fluid droplets (cf. figure 2). However value of this yield stress threshold is low enough to have no effects on the ulterior behavior of the droplet. Yet the initial shape of the droplet is close to a prolate spheroid whose length to width ratio equals 2 (up to $4 \%$ deviation).

Table 1. Liquid properties.

\begin{tabular}{cccccc}
\hline & $\square$ & $k$ & $\mu$ & $C_{p}$ & $H_{f}$ \\
\cline { 2 - 6 } & kg.m ${ }^{-3}$ & $\mathrm{~W} /(\mathrm{m} . \mathrm{k})$ & $\mathrm{Pa} . \mathrm{s}$ & $\mathrm{J} /(\mathrm{Kg} \cdot \mathrm{K})$ & $\mathrm{J} / \mathrm{Kg}$ \\
\cline { 2 - 6 } Field Metal & 7994 & 5.44 & 0.01 & 300 & 26415 \\
\cline { 2 - 6 } Water & 997 & 0,6071 & 0.001 & 4185 & 334000 \\
\hline
\end{tabular}



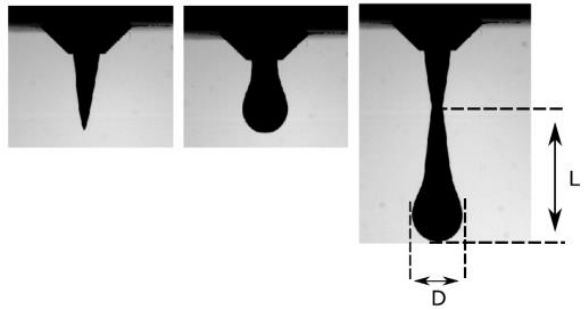

Figure 2. Liquid Metal Droplet Pinch-Off.

Experiments range over six different Weber number and 3 bath temperatures $\left(28^{\circ} \mathrm{C}, 40^{\circ} \mathrm{C}\right.$ and $\left.50^{\circ} \mathrm{C}\right) . A$ fter $50^{\circ} \mathrm{C}$, fragments remains liquid long enough to merge in a pool and cannot be collected anymore. Initial temperature and mass of the droplets are kept constant at, respectively, $85^{\circ} \mathrm{C}$ and $0.27 \mathrm{~g}\left(\mathrm{D}_{0}=4 \mathrm{~mm}\right)$. Initial velocity of the droplet range from $0.5 \mathrm{~m} / \mathrm{s}$ à $3.5 \mathrm{~m} / \mathrm{s}$ according to their initial height above the bath (cf. table 2). As drag effects are negligible over such small heights, their impact velocity can be estimated to be

$$
U=\sqrt{2 g z}
$$

Table 2. Different Weber numbers tested.

\begin{tabular}{llll}
\hline We & $z(\mathrm{~cm})$ & $U(\mathrm{~m} / \mathrm{s})$ & $R e$ \\
\hline 118 & 60 & 3.43 & 13809 \\
\hline 99 & 50 & 3.13 & 12606 \\
\hline 79 & 40 & 2.80 & 11275 \\
\hline 59 & 30 & 2.42 & 9765 \\
\hline 39 & 20 & 1.98 & 7973 \\
\hline 20 & 10 & 1.40 & 5637
\end{tabular}

Time will be non dimensionalized with the Ranger and Nicholls [3] characteristic atomization time given by

$$
\begin{aligned}
& t_{R N}=\frac{U_{0}\left(\frac{\rho_{L}}{\rho_{A}}\right)^{0.5}}{D_{0}} . \\
& t^{*}=\frac{t}{t_{R N}}
\end{aligned}
$$

\section{Results}

Figure 3 shows the impact of the droplet on the water surface. On such small height (i.e. less than 60 $\mathrm{cm})$, neither large water deformation nor air entrainment is observed.

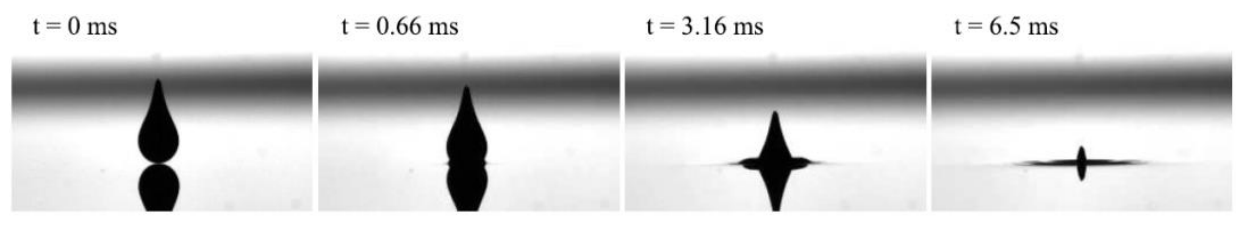

Figure 3. Liquid Metal Droplet Impact on the water surface

Figure 4 depicts the ulterior behaviour of the droplet. According to the Weber number the droplet can either oscillate or deform into a spherical bag (whose concavity is opposite to the gas-liquid case). Fragmentation mechanism is therefore very similar to classical bag breakup in gas liquid case except the reversed concavity of the bag. This may be related to the production of an intense vortex ring in the wake of the droplet (CastrillonEscobar [9]). Rayleigh-Taylor waves can be observed but the breakup of the bag is closer to the bursting of soap bubble as can be seen on figure 4 . This produces very small droplets while larger ligaments eventually destabilize to produce larger droplets leading to a multi-peaked distribution. 

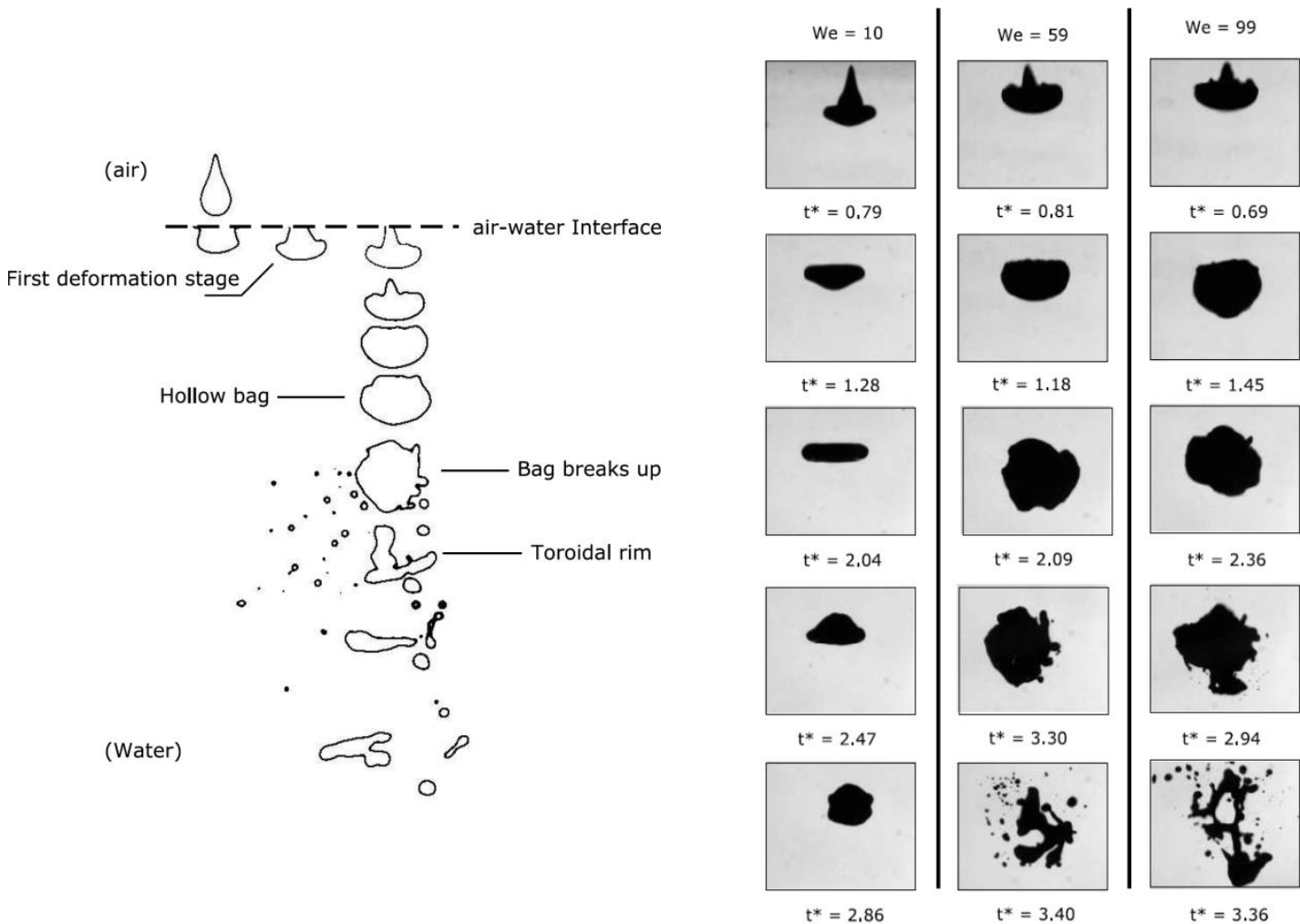

Figure 4. Deformation and fragmentation of the droplet without solidification. $T_{L}=85^{\circ} \mathrm{C} \cdot T_{A}=50^{\circ} \mathrm{C}$.

According to the Weber number, different behaviors are observed. For We $<12$, droplet oscillates between an oblate and a prolate shape while for $12<\mathrm{We}<20$ this oscillation may produce fragmentation when oscillating in the prolate shape. After We $>20$ and up to the maximum tested value of 120 , the droplet deforms into a spherical hollow cap which can eventually be solidified when the water temperature is cold enough (cf. figure 5). This bubble can then burst leading to a population of fine droplet and large ligaments threaded into cobweb like structures.
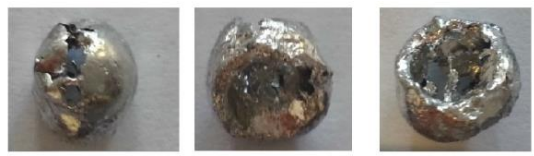

Figure 5. Liquid Metal Droplet Frozen during the reverse bag formation. $T_{A}=20^{\circ} \mathrm{C}$

Thought not detailed in present paper, figure 6 shows a comparison between a DNS obtained using the Basilisk solver and present experiments. Lambda 2 criterion is used to visualize the vortex ring that appear in the wake of the droplet (cf. Castrillon Escobar [9]). That criterion looks for the eigenvalue of the tensor $\mathrm{S}^{2}+\Omega^{2}$ which can be considered as a good approximation of the pressure Hessian. Zones where the second eigenvalue is negative $\left(\lambda_{2}<0\right)$ are likely to be low pressure region owing to the presence of a vortex. As the density ratio between droplet and surrounding liquid is lower than in the gas liquid case, the inertia created by this rotary motion can strongly affect the droplet shape. This seems in agreement with early 2D DNS results obtained by (Han and Tryggvasson, [10]) who however did not look for these vortices. 


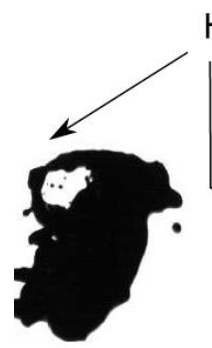

Hole growth

$0 \mathrm{~ms}$

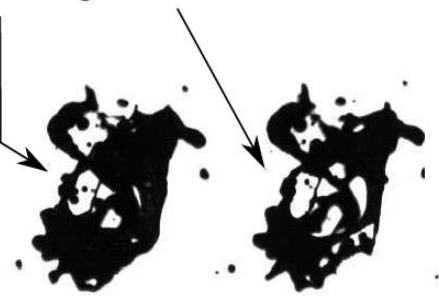

$2 \mathrm{~ms}$

$2.5 \mathrm{~ms}$ $t^{*}=0$

$t^{*}=0.63$

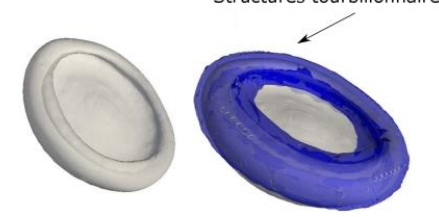

$\mathrm{t}^{*}=0.95$

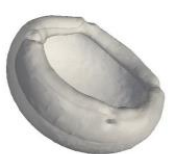

Ligaments Small fragments

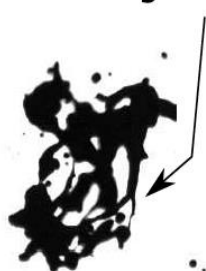

$3 \mathrm{~ms}$

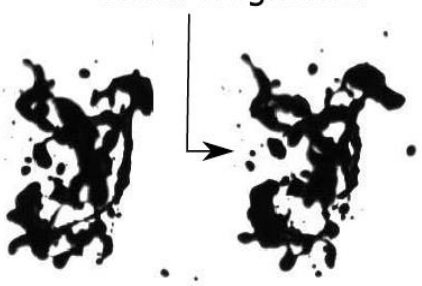

$4 \mathrm{~ms}$

$5 \mathrm{~ms}$

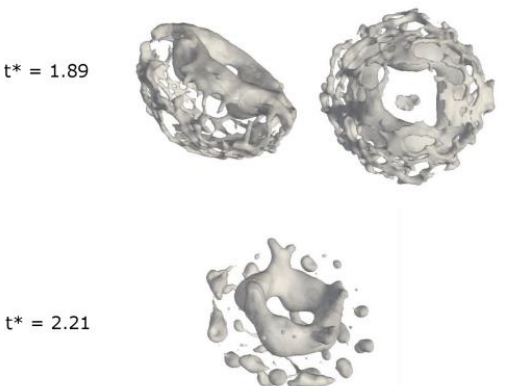

Flow $\uparrow \uparrow \uparrow \uparrow$

Figure 6. Comparison between experimental results and numerical simulations with Basilisk.

Figure 7 shows the values of measured Sauter Mean Diameter (SMD) and Mass Mean Diameter (MMD) after sieving and weighting the solidified fragments (the sieve sizes are 20,50, 100, 500,1000 and $2000 \mu \mathrm{m}$ ). For Weber number 20, the droplet fragments during its prolate elongation generating two main daughter droplets of roughly equal shape, therefore $S M D / D_{0}$ ratio equals $1 / 2$. Ratio between $S M D$ and MMD range from 1 to 1,5 in agreement with DNS results of Jalaal and Mehravaran [11]. As Weber number increases these average size of fragments decreases and it can also be seen that, as expected, the colder the water, the larger the fragments. A possible interpretation to these graphs is that, as surrounding temperature decreases, a larger solidified crust can form at the interface between liquid metal and water. This crust will then increase the apparent surface tension of the droplet. Therefore the real Weber number should be lower owing to this increased surface tension and the corrected curve should shift to the left. This will be the focus of next section.
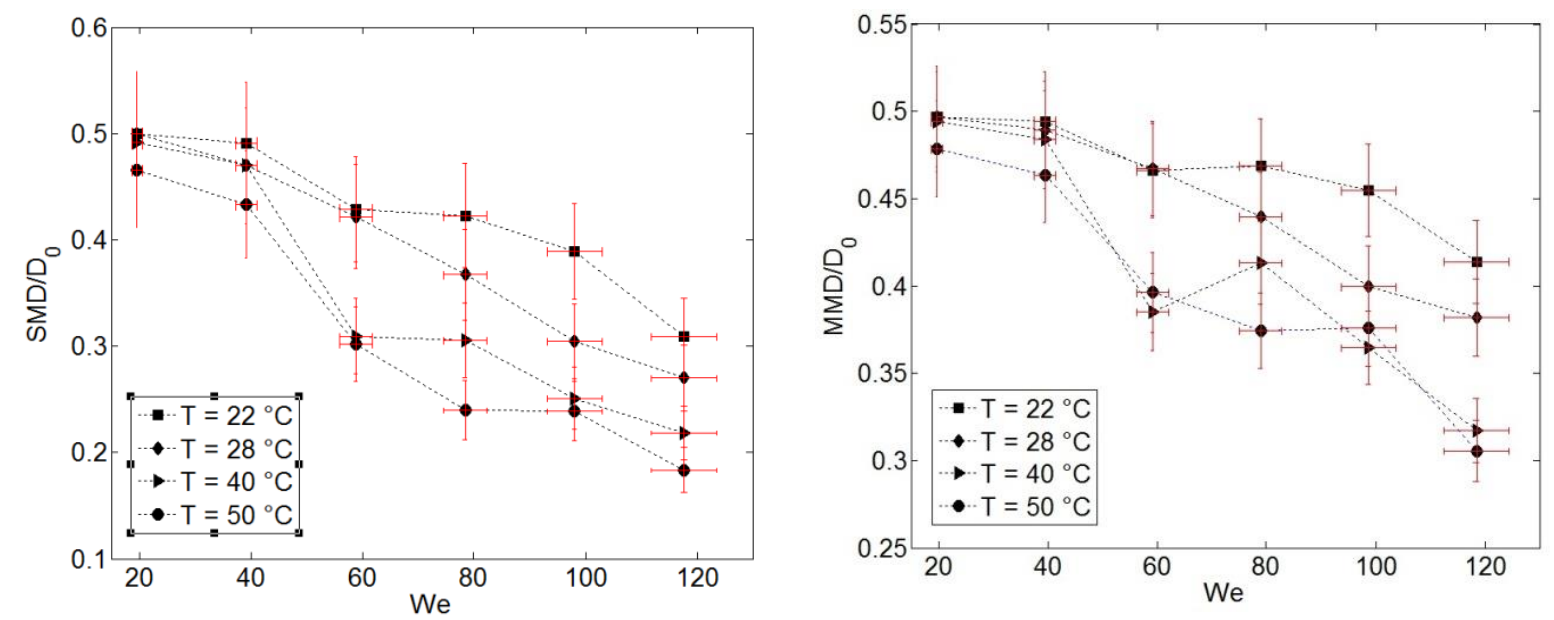

Figure 7. Sauter Mean Diameter and Mass Mean Diameter of the solidified fragments as a function of Weber number. 


\section{Discussion}

Owing to the previous results, it seems to be interesting to find a model that can account for the shift to the right of the curve as the bath temperature is decreased. Haraldsson et al. [6] gave us a hint as they devised a simple model for the crust growth and used it to see how it could affect the growth rate of Kelvin-Helmholtz instability. However this crust does grow over time, therefore a specific time instant must be selected to create a new Weber number. In the present work, it has been decided to use the fragmentation inception time given by eq. 7.

$$
t_{\text {frag }}=8(W e-12)^{-0.25}\left(1+2.2 O h^{1.6}\right) t_{R N}
$$

In the following the droplet is assumed to be still spherical and the crust thickness $\delta$ is assumed to be thin. The solidification time is supposed to be the sum of the time needed to remove the sensible heat from the droplet and the time needed for the crust to grow.

$$
t_{\text {sol }}=t_{\text {sup }}+t_{\text {fus }}, \quad t_{\text {sup }}=\frac{\rho_{L} C p_{L} \Delta T_{\text {sup }} \delta}{q^{\prime \prime}}, t_{\text {fus }}=\frac{\rho_{L} H f_{L} \delta}{q^{\prime \prime}}
$$

The heat flux $q$ " is obtained from a classical correlation for a sphere:

$$
q^{\prime \prime}=\frac{k_{A}}{D_{0}}\left(2+0.6 \operatorname{Re}^{0.5} \operatorname{Pr}^{1 / 3}\right)\left(T_{L}-T_{A}\right)
$$

Therefore crust thickness at time $t_{\text {frag }}$ is given by

$$
\delta\left(t_{\text {frag }}\right)=\frac{t_{\text {frag }} q^{\prime \prime}}{\rho_{L}\left(C p_{L} \Delta T_{\text {sup }}+H f\right)} .
$$

This thin solidified shell is elastic and possesses a Young modulus $E$ of $9.25 \mathrm{GPa}$ (cf. Shan et al. [12]). Following Haraldsson et al. [6], it is possible to convert the elastic energy of deformation into a surface energy thanks to

$$
\sigma_{\text {elas }}=\frac{E \delta^{3}}{12\left(1-v^{2}\right)^{3} D_{0}^{3}}
$$

where $v$ is Poisson ratio (taken to be $1 / 3$ ). Lastly, the new Weber number is

$$
W e_{a p p}=\frac{\rho_{A} D_{0} U_{L}^{2}}{\sigma_{a p p}}
$$

where apparent surface tension is the sum of the liquid surface tension and of this elastic surface tension

$$
\sigma_{\text {app }}=\sigma+\sigma_{\text {elas }}
$$

Figure 7 shows the evolution of the ratio $S M D / D_{0}$ as a function of this new Weber number. As expected all curves are shifted to the left and seem to collapse on a common master curve.

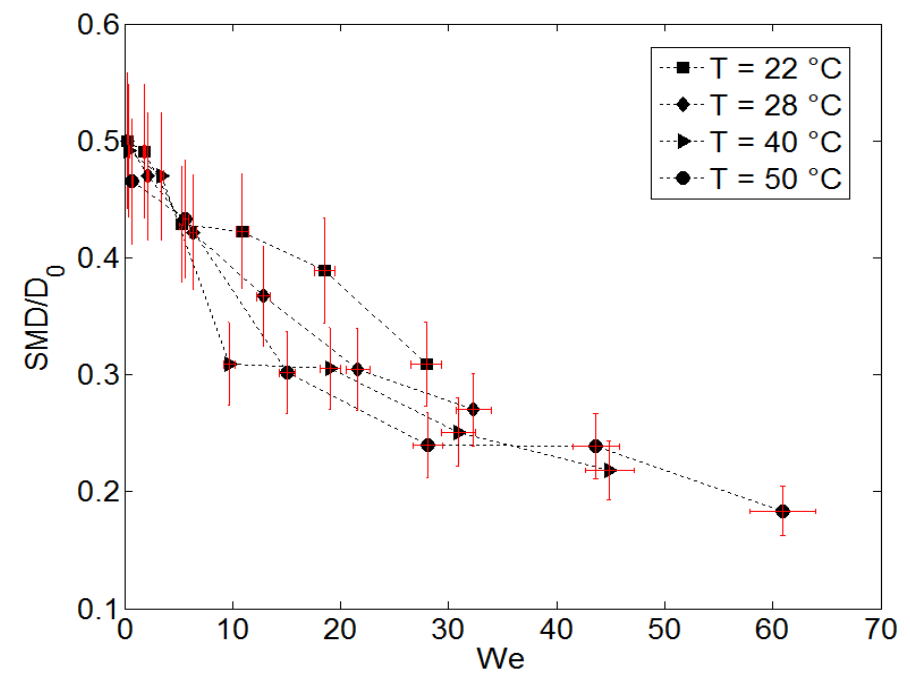

Figure 8. Sauter Mean Diameter of the solidified fragments as a function of the modified Weber number. Initial droplet temperature is $85^{\circ} \mathrm{C}$. 


\section{Conclusions}

In this work, a single heated liquid metal droplet impact into cold water has been studied thanks to a specifically designed experimental setup. The temperature of the water bath has been increased from $20^{\circ} \mathrm{C}$ to $50^{\circ} \mathrm{C}$. The initial temperature of the droplet has been kept constant $85^{\circ} \mathrm{C}$ way above the liquid metal solidification point $62^{\circ} \mathrm{C}$ ). For the lowest value of the bath temperature $\left(20^{\circ} \mathrm{C}\right)$ and for high enough value of the Weber number (We>20), the droplet can be frozen before its breakup during its initial deformation into a hollow shell showing that this experiment is well designed to study the interplay between solidification and fragmentation. For the higher value of the water temperature, different fragmentation regimes have been put into evidence: below $\mathrm{We}<12$, the droplet does not fragment and oscillates. Between $12<\mathrm{We}<20$, the droplet oscillates and fragments during the prolate part of its oscillation. It mainly produces two daughter droplets of equal size. Bag breakup happens when We $>20$, but the concavity of the bag is opposite to the liquid-gas case owing to the strong influence of the vortex ring that is produced in the wake of the droplet by flow separation. The liquid metal bubble then bursts and produces a mixture of fine droplets and a cobweb of ligaments which then fragments into bigger droplets. As the temperature of the bath is lowered, the size of the fragments increases. This can be related to a stronger influence of solidification on the fragmentation mechanism. A simple way to account for this is to create a new Weber number which sums up the surface tension and the elastic energy of the solidified crust at the inception of the droplet fragmentation. This allows the different curves (SMD and MMD as a function of the droplet Weber number for the different bath temperature) to collapse on a common master curve.

\section{Acknowledgements}

This work has been funded by French Government through "programme d'investissements d'avenir" AnR RSNR ICE under the grant $n^{\circ}$ ANR-10-RSNR-01. The authors would also like to mention support from EU-COST Action MP1305 "Flowing Matter".

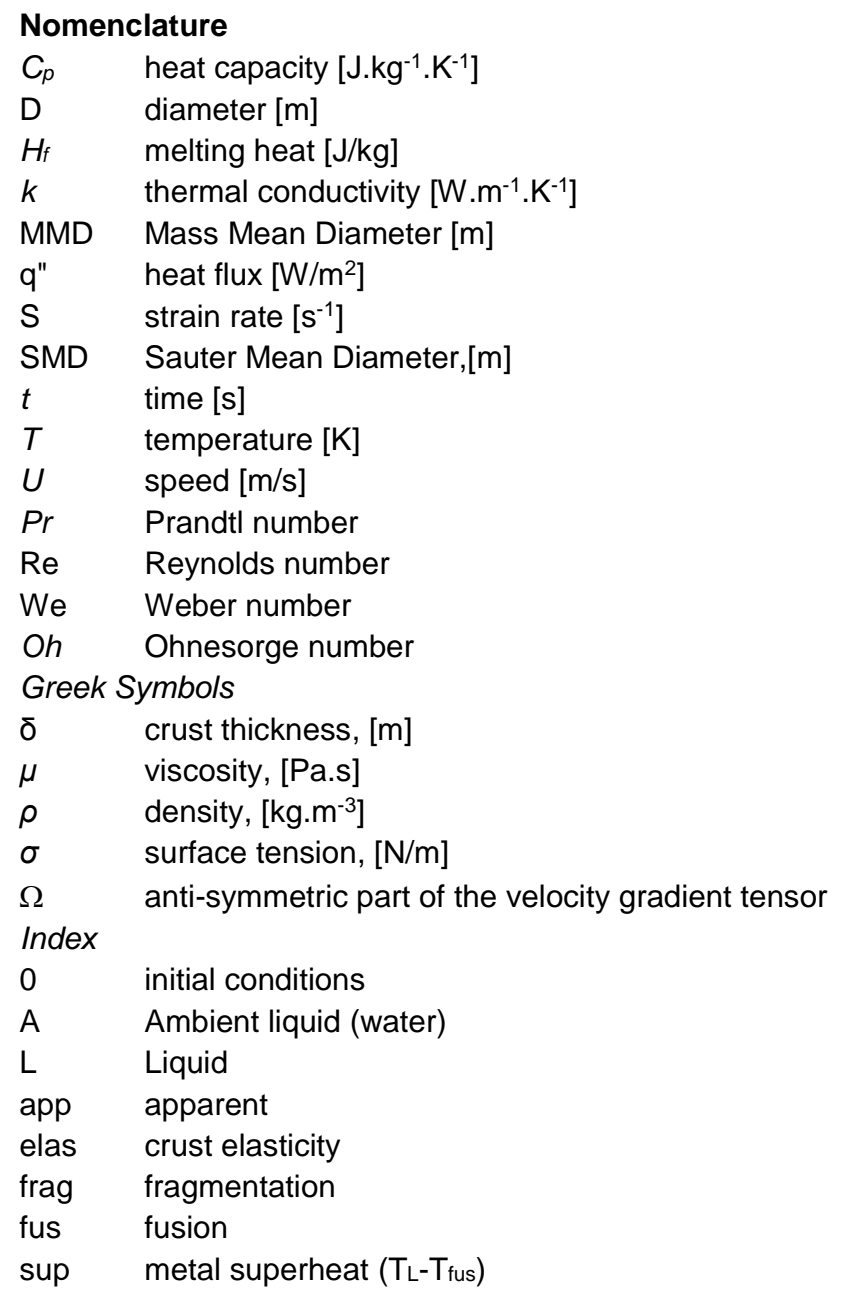




\section{References}

[1] Hsiang and Faeth, (1995) Int. J. Multiph. Flow 21, 545.

[2] Landeau et al., (2014) J. Fluid Mech. 749, 478.

[3] Ranger and Nicholls (1969), AIAA J. 7, 285.

[4] Gelfand (1996), Prog. Energy Combust. Sci. 22, 201.

[5] Kim et al., (1983) in Proc. Int. Meet. Light Water Sev. Accid. Eval., edited by American Nuclear Society (Stone \& Webster Engineering Corp,), p. 4.1-4.7.

[6] Haraldsson et al., (2001) Heat Mass Transf. 37, 417.

[7] Popinet, (2003) J. Comput. Phys. 190, 572.

[8] Xu et al., (2012) Phys. Fluids 24, 63101.

[9] Castrillon Escobar (2016), Ph.D. Thesis, Univ. Lorraine.

[10] Han and Tryggvason, (2001), Phys. Fluids 13(6), 1554

[11] Jalaal and Mehravaran (2012), Int. J. Multiph. Flow 47, 115.

[12] Shan et al. (2013), Smart Mater. Struct. 22, 85005. 